\title{
A case of recurrent fallopian tube carcinoma in situ with para-aortic lymph node metastasis
}

\author{
Hidetaka Nomura Akiko Abe $\cdot$ Akiko Yamamoto $\cdot$ Maki Matoda \\ Kohei Omatsu $\cdot$ Kazuyoshi Kato $\cdot$ Kenji Umayahara $\cdot$ Reiko Furuta • \\ Nobuhiro Takeshima
}

Received: 27 August 2012 / Accepted: 8 January 2013/Published online: 26 January 2013

(C) The Japan Society of Clinical Oncology 2013

\begin{abstract}
We encountered a case of recurrent fallopian tube carcinoma in situ with para-aortic lymph node metastasis 9 months after the first surgery. The patient presented with genital bleeding, and endometrial cytology revealed extrauterine malignant cells. However, endometrial biopsy and imaging modalities could not detect any abnormalities. We performed a total abdominal hysterectomy and bilateral salpingo-oophorectomy after obtaining the patient's consent. Histopathological examination revealed atypical cells in the fimbrial portion of the fallopian tube. A diagnosis of fallopian tube carcinoma in situ was made. No further adjuvant treatment was administered. Nine months later, fluorine-18 fluorodeoxyglucose positron emission tomography/computed tomography revealed pathologic activity in the para-aortic lymph node and CA125 elevation. A diagnosis of metastatic adenocarcinoma was established on the basis of histological examination of a frozen section. The patient underwent para-aortic and pelvic lymph node dissection and omentectomy. The pathology report revealed metastasis to 3 of 69 lymph nodes; however, peritoneal cytology was negative for cancer. To the best of our knowledge, this is the first reported case of recurrent fallopian tube carcinoma in situ.
\end{abstract}

H. Nomura $(\bowtie) \cdot$ A. Abe · A. Yamamoto · M. Matoda .

K. Omatsu - K. Kato - K. Umayahara - N. Takeshima

Department of Gynecology, Cancer Institute Hospital,

Ariake 3-8-31, Koto-ku, Tokyo 135-8550, Japan

e-mail: hidetaka.nomura@jfcr.or.jp

R. Furuta

Division of Pathology, The Cancer Institute, Japanese

Foundation for Cancer Research, Ariake 3-8-31, Koto-ku,

Tokyo 135-8550, Japan
Keywords Fallopian tube carcinoma in situ - Para-aortic lymph node metastasis · Tubal carcinoma

\section{Introduction}

The study of early events in ovarian carcinogenesis is hampered because more than $80 \%$ ovarian cancers have already metastasized beyond the ovary at the time of diagnosis. In the last decade, observations of women undergoing risk-reducing (prophylactic) salpingo-oophorectomy for hereditary breast and ovarian cancer (HBOC) syndrome have increased the understanding of early ovarian cancer. Careful sectioning of fallopian tubes from riskreducing salpingo-oophorectomy specimens frequently reveals areas of marked cytologic atypia and disorganized growth within the fimbria. These areas have been termed carcinoma in situ or tubal dysplasia [1,2].

The patient whose case is reported here had no familial history of HBOC, but was surgically diagnosed with fallopian tube carcinoma in situ. No further adjuvant treatment was administered. However, 9 months later, para-aortic lymph node metastasis was detected. This is the first reported case of recurrent fallopian tube carcinoma in situ.

\section{Case report}

A 39-year-old G:2 P:2 woman presented with atypical genital bleeding. She had no family history of ovarian or breast cancer. Endometrial cytology showed malignant cells surrounded by normal endometrial cells. Extrauterine malignancy was suspected. Endometrial biopsy and fluorine-18 fluorodeoxyglucose positron emission tomography/ 
computed tomography (PET/CT) could not detect any abnormalities. Therefore, she was referred to our hospital.

Endometrial biopsy and cytology analysis were performed to confirm the malignancy; however, no malignant cells were detected. Transvaginal ultrasound, pelvic exam, MRI, and CA125 findings were normal. Because we could not deny the existence of fallopian tube carcinoma, a total abdominal hysterectomy and bilateral salpingo-oophorectomy were performed after obtaining the patient's consent. Intraoperative evaluation revealed no abnormalities. Cytology samples obtained from peritoneal washing were negative for cancer, and macroscopically no obvious tumor was detected in both adnexa. However, careful sectioning (every $4 \mathrm{~mm}$ ) of both ovaries and fallopian tubes (Fig. 1) revealed an area of cytologic atypia and disorganized growth within the left fimbria. No atypical cells were detected on the opposite side of the sample section. Deep sectioning of the original slide revealed carcinoma in situ measuring $\leq 0.5 \mathrm{~mm}$ in size (Figs. 2, 3). The carcinoma in situ was only detected in two of the four deep section slides. These cells showed nuclear atypia manifested in the form of nuclear enlargement, hyperchromasia, and prominent nucleoli. We attempted p53 staining, but no atypical cells were detected on the deep section slides and no other areas were stained. The diagnosis of fallopian tube carcinoma in situ was confirmed. No further adjuvant treatment was administered.

Nine months later, routine transabdominal ultrasound revealed an enlarged para-aortic lymph node. PET/CT revealed pathologic activity only in the para-aortic lymph node $\left(\mathrm{SUV}_{\max }, 2.42\right)$ (Figs. 4, 5), and no other metastasis was detected. The patient's CA125 level was $42.7 \mathrm{IU} / \mathrm{ml}$. Recurrent fallopian tube carcinoma was suspected, for which the patient underwent further surgery. A diagnosis of metastatic adenocarcinoma was established on the basis of histological examination of a frozen section. The patient

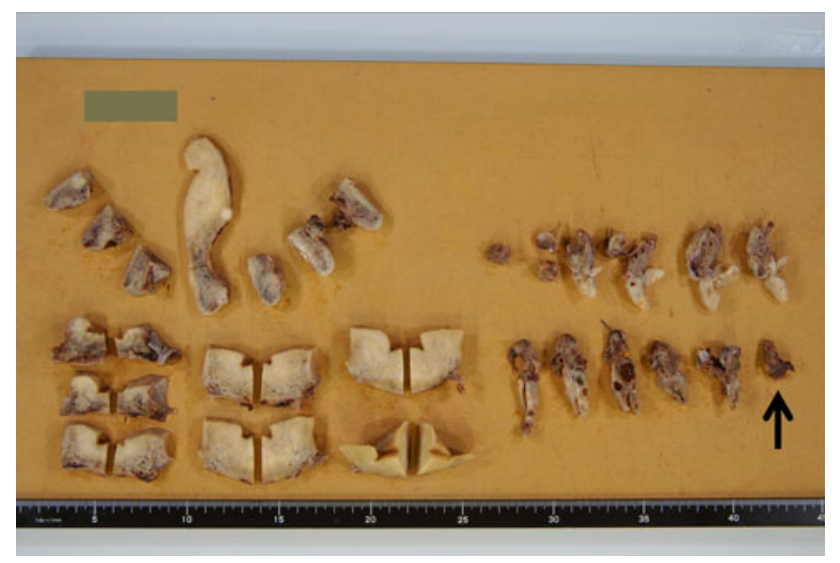

Fig. 1 Sectioning of the sample. Arrow shows the left fimbria in which carcinoma in situ was detected

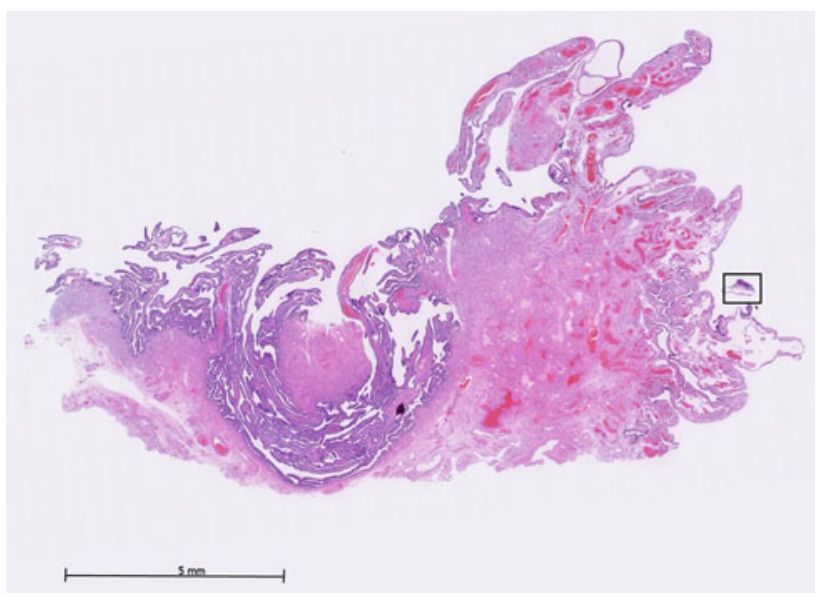

Fig. 2 Carcinoma in situ was detected in the left fimbria (square)

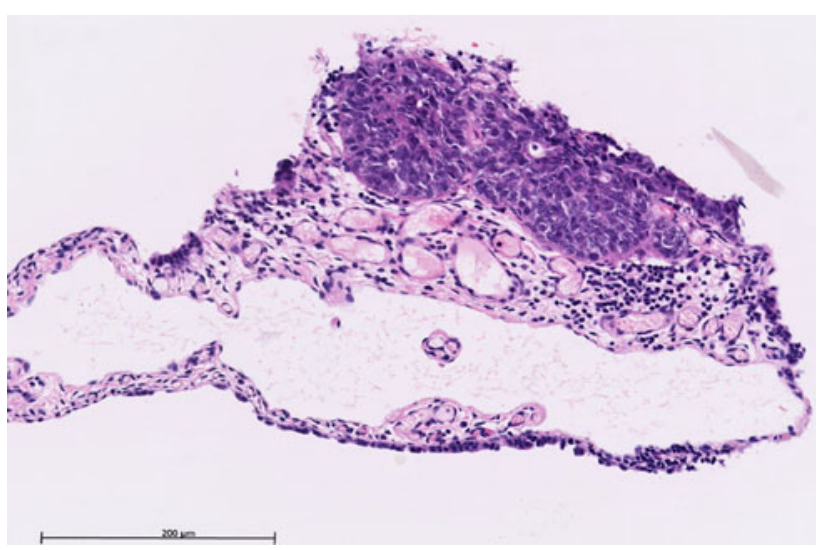

Fig. 3 Nuclear atypia manifested in the form of nuclear enlargement, hyperchromatia, and prominent nucleoli

subsequently underwent para-aortic and pelvic lymph node dissection and omentectomy. The pathological diagnosis for the recurrent tumor was adenocarcinoma. And the pathology report revealed metastatic adenocarcinoma in 3 of 69 lymph nodes. No cancer cells were detected in the omentum. Peritoneal cytology was negative for cancer. The patient is currently undergoing chemotherapy (paclitaxel: $175 \mathrm{mg} / \mathrm{m}^{2}$, carboplatin: AUC 6).

\section{Discussion}

Fallopian tube carcinoma is one of the rarest malignancies of the female genital tract. It constitutes $<1 \%$ of all gynecologic cancers, with an average annual incidence of 3.3 per 1,000,000 women in the USA. Factors associated with the increase in the incidence of the malignancy include a decrease in parity, higher socioeconomic status, pelvic inflammatory disease, improved diagnosis, and increased longevity. Fallopian tube carcinoma has been associated with BRCA mutations in several recent case 


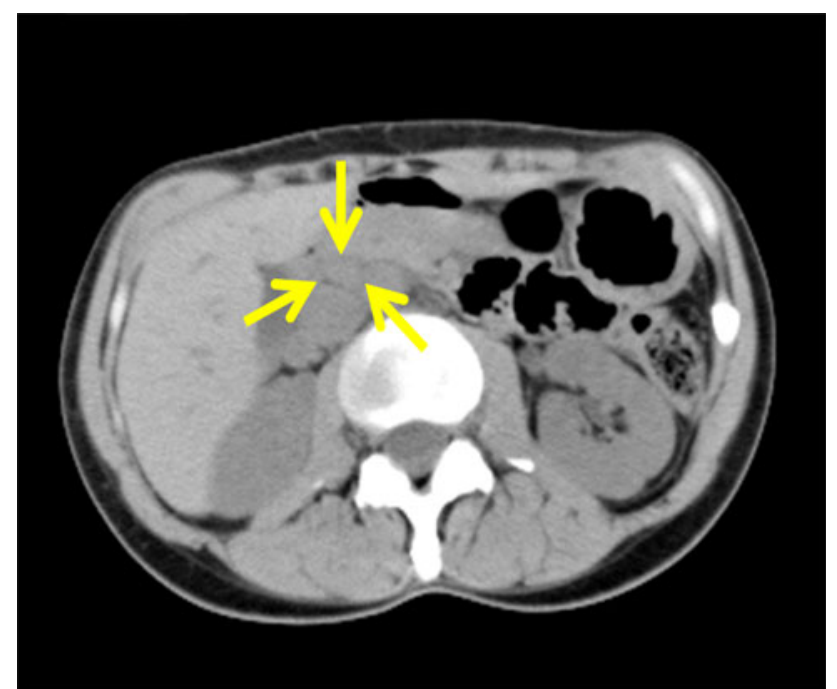

Fig. 4 Swollen para-aortic lymph node (arrows)

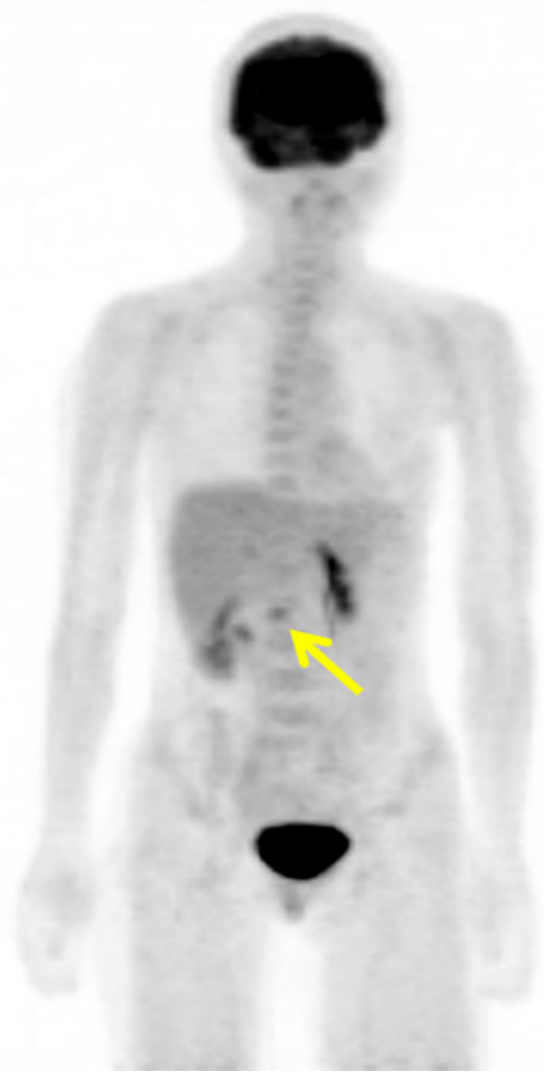

Fig. 5 FDG accumulation at the para-aortic lymph node (arrow)

series, and its incidence in this subset of women is reported to be as high as $3 \%$, which is an estimated 120-fold increase in risk compared with the general population [3].

Crum et al. [4] reported that careful sectioning of fallopian tubes from risk-reducing salpingo-oophorectomy specimens frequently reveals areas of marked cytologic atypia and disorganized growth within the fimbria. These areas have been termed carcinoma in situ or tubal dysplasia $[1,2]$ and are characterized by positive p53 immunostaining (which correlates with mutations in the TP53 gene), abnormal proliferation, and DNA damage [5]. Approximately $5 \%$ of women undergoing risk-reducing surgery are diagnosed with an occult ovarian cancer (mostly high-grade serous carcinoma) [6, 7]. Furthermore, approximately one-third of morphologically normal fimbriae from women without a hereditary ovarian cancer risk exhibit areas of p53 staining without atypia or abnormal proliferation (termed the p53 signature) $[5,8,9]$. Collectively, these observations have led to the hypothesis that most serous carcinomas that are clinically classified as ovarian or peritoneal may in fact arise from the epithelium of the fallopian tube. However, given that much of this research has been performed on archival paraffin-embedded tissues, direct testing of this hypothesis has proven difficult [10]. Carcangiu et al. [11] reported atypical hyperplasia or dysplasia of the fallopian tube epithelium in 2 of $22(10 \%)$ specimens from high-risk women undergoing risk-reducing salpingo-oophorectomy, and Piek et al. [12] reported the same condition in 6 of $12(50 \%)$ such women. Piek et al. also reported atypical hyperplasia, considered to be less severe than dysplasia, in another 5 of 12 patients. Przybycin et al. reported that $59 \%(24 / 45)$ of high-grade pelvic (non-uterine) serous carcinomas are associated with fallopian tube carcinoma in situ. This is consistent with the hypothesis that the fallopian tube cells are the source of the majority of these tumors [13]. It was considered to be extremely rare for fallopian tube carcinoma in situ to be detected in women without high-risk factors for ovarian cancer. In this case, although the patient presented with atypical genital bleeding, she was nursing at that time. Genital bleeding may be caused by hormonal dysfunction.

According to Manchanda et al. [14], there were no recurrences in their 19 patients with fallopian tube carcinoma in situ. Powell et al. [15] reported five patients with fallopian tube carcinoma in situ, but none showed any evidence of remission. Management of primary fallopian tube cancer is generally similar to that of ovarian cancer, and comparable 5-year survival rates have been reported for stage 1a and stage $1 \mathrm{~b}$ ovarian and fallopian tube cancers. Some advise adjuvant chemotherapy (carboplatin and paclitaxel) for stage 1c (any grade) or high-grade (grade 3) stage 1a and stage $1 \mathrm{~b}$ disease [16]. The presence of positive cytology would thus affect the management of grade $1 / 2$ stage $1 \mathrm{a} /$ stage $1 \mathrm{~b}$ fallopian tube or ovarian cancers. However, some authorities advocate that chemotherapy should be considered for all stage 1 fallopian tube cancers [17]. Because the fallopian tube lumen directly communicates with the peritoneal cavity, researchers propound that stage 
1a fallopian tube cancer has a higher predisposition for distant microscopic spread and is functionally equivalent to stage 1c ovarian cancer. However, there have been no reports recommending adjuvant therapy for fallopian tube carcinoma in situ.

Koo et al. [18] studied the efficacy of para-aortic lymphadenectomy for invasive primary fallopian tube cancer. Four $(26.7 \%)$ of their patients had nodal involvement, two with para-aortic lymph node involvement and two with both pelvic and para-aortic lymph node involvement. None of the patients was positive for pelvic lymph nodes alone. They highlighted the importance of comprehensive paraaortic lymphadenectomy for accurate staging of primary fallopian tube cancer. There have been some reports of distant isolated metastasis from fallopian tube cancer such as in the supraclavicular lymph node, cardiophrenic angle lymph node, and sigmoid colon [19-21]. It is important to bear in mind that distant metastasis may occur after treatment of fallopian tube cancer.

When malignant cells are limited to the fimbria and no invasion has been detected, most clinicians may not consider any other adjuvant therapy. We have presented a case of recurrent fallopian tube carcinoma in situ with paraaortic lymph node metastasis. More cases of fallopian tube carcinoma in situ need to be reviewed, and whether adjuvant therapy is required must be evaluated. Further reports are expected.

Conflict of interest The authors declare that they have no conflict of interest.

\section{References}

1. Piek JM, Verheijen RH, Kenemans $P$ et al (2003) BRCA1/2related ovarian cancers are of tubal origin: a hypothesis. Gynecol Oncol 90:491

2. Lee Y, Miron A, Drapkin R et al (2007) A candidate precursor to serous carcinoma that originates in the distal fallopian tube. J Pathol 211:26-35

3. DiSaia PJ, Creasman WT (2007) Clinical gynecologic oncology. Mosby, Philadelphia

4. Crum CP, Drapkin R, Kindelberger D et al (2007) Lessons from BRCA: the tubal fimbria emerges as an origin for pelvic serous cancer. Clin Med Res 5:35-44

5. Folkins AK, Jarboe EA, Saleemuddin A et al (2008) A candidate precursor to pelvic serous cancer (p53 signature) and its prevalence in ovaries and fallopian tubes from women with BRCA mutations. Gynecol Oncol 109:168-173

6. Lamb JD, Garcia RL, Goff BA et al (2006) Predictors of occult neoplasia in women undergoing risk-reducing salpingo-oophorectomy. Am J Obstet Gynecol 194:1702-1709

7. Laki F, Kirova YM, This P et al (2007) Prophylactic salpingooophorectomy in a series of 89 women carrying a BRCA1 or a BRCA2 mutation. Cancer 109:1784-1790

8. Norquist BM, Garcia RL, Allison KH et al (2010) The molecular pathogenesis of hereditary ovarian carcinoma: alterations in the tubal epithelium of women with BRCA1 and BRCA2 mutations. Cancer 116:5261-5271

9. Shaw PA, Rouzbahman M, Pizer ES et al (2009) Candidate serous cancer precursors in fallopian tube epithelium of BRCA1/ 2 mutation carriers. Mod Pathol 22:163-167

10. Jazaeri AA, Bryant JL, Park H et al (2011) Molecular requirements for transformation of fallopian tube epithelial cells into serous carcinoma. Neoplasia 13:899-911

11. Carcanigu ML, Radice P, Manoukian S et al (2004) Atypical epithelial proliferation in fallopian tubes in prophylactic salpingo-oophorectomy specimens from BRCA1 and BRCA2 germline mutation carriers. Int J Gynecol Pathol 23:35-40

12. Piek JM, van Diest PJ, Zweemer RP et al (2001) Dysplastic changes in prophylactically removed Fallopian tubes of women predisposed to developing ovarian cancer. J Pathol 195:451-456

13. Przybycin CG, Kurman RJ, Ronnett BM et al (2010) Are all pelvic (non-uterine) serous carcinomas of tubal origin? Am J Surg Pathol 34:1407-1416

14. Manchanda R, Drapkin R, Jacobs I et al (2012) The role of peritoneal cytology at risk-reducing salpingo-oophorectomy (RRSO) in women at increased risk of familial ovarian/tubal cancer. Gynecol Oncol 124:185-191

15. Powell CB, Chen LM, McLennan J et al (2011) Risk-reducing salpingo-oophorectomy (RRSO) in BRCA mutation carriers: experience with a consecutive series of 111 patients using a standardized surgical-pathological protocol. Int J Gynecol Cancer 21:846-851

16. Vaysse C, Touboul C, Filleron T et al (2011) Early stage (IA-IB) primary carcinoma of the fallopian tube: case-control comparison to adenocarcinoma of the ovary. J Gynecol Oncol 22:9-17

17. Pectasides D, Pectasides E, Papaxoinis G et al (2009) Primary fallopian tube carcinoma: results of a retrospective analysis of 64 patients. Gynecol Oncol 115:97-101

18. Koo YJ, Kwon YS, Lim KT et al (2011) Para-aortic lymphadenectomy for primary fallopian tube cancer. Int J Gynaecol Obstet 112:18-20

19. Sakurai N, Tateoka K, Fukaya K et al (2010) Supraclavicular lymph node metastasis as the initial presentation of primary fallopian tube carcinoma. Int J Clin Oncol 15:301-304

20. Eguchi T, Takasuna K, Nakayama A et al (2012) Cardiophrenic angle lymph node metastasis from a fallopian primary tumor. Asian Cardiovasc Thorac Ann 20:74-76

21. Deliveliotou A, Fotiou S, Karvouni E et al (2011) Isolated sigmoid colon metastasis from a primary fallopian tube carcinoma: a case report. Eur J Gynaecol Oncol 32:585-587 